\title{
How specialist nurse-led care can help to lower the costs of prophylaxis
}

Allison Greig

Prophylaxis with factor VIII concentrates is beneficial for adults with severe haemophilia $A$, but prophylaxis regimens are costly. Guidelines recommend personalised prophylaxis tailored to bleeding phenotype and the result of pharmacokinetic assessments. This article describes a project in which specialist nurses played a pivotal role in identifying a small cohort of patients eligible for dose reduction. Nurses monitored patients following dose reduction, maintaining contact in order to address fears and ensure patient safety. The project led to a substantial cost saving for the Trust and demonstrates the value that nurses can bring to the haemophilia centre.

It is well recognised that prophylaxis is beneficial for people with severe haemophilia A. UKHCDO treatment guidelines suggest that prophylaxis regimens in adults with severe haemophilia A should be personalised, based on bleeding phenotype and pharmacokinetic studies [1]. Published data suggest that the half-life of infused factor VIII concentrates ranges from 8 to 23 hours $[2,3]$.

Collins [4] recommends individually tailored treatment regimens, agreed between the patient and their haemophilia centre. He further recommends that these regimens be reviewed regularly as an individual's pharmacokinetics may vary with age and lifestyle factors and may change with the season.

The Haemostasis and Thrombosis Centre at St Georges Healthcare NHS Trust in London cares for approximately 600 patients with inherited bleeding disorders (IBD). These include 40 patients with severe haemophilia A, of whom 30 are adults and 10 children. In 2013, the total cost of factor VIII concentrates delivered to patients for home treatment was in the region of $£ 4.6$ million. Prophylaxis regimens are costly and haemophilia centre staff have an obligation to ensure that resources are used appropriately.

We reviewed all prophylaxis regimens in adults with severe haemophilia $A$, in order to ensure that all patients were prescribed the minimum effective dose. We defined the minimum effective dose as factor VIII trough level of 1-3 IU/dL and less then three spontaneous bleeds in six months. We believed that this approach could offer significant economic benefit while maintaining clinically effective treatment that is acceptable to patients.

Allison Grieg, Clinical Nurse Specialist, St George's Healthcare NHS Trust Blackshaw Road, London SW17 OQT

Email: Allison.Greig@astgeorges.nhs.uk

\section{Methods}

We identified 26 patients, within our cohort, who were infusing factor VIII concentrates every 48 hours. The dose infused ranged from 14-49 IU/kg. Patients attending their routine clinic review were asked to infuse their usual prophylaxis dose 48 hours prior to their appointment. Ten patients were unable to provide a trough sample, either because treatment had been administered within the preceding 48 hours, or because the patient did not attend clinic. Patients were also reminded to update their treatment calendars, either electronically or using paper log sheets.

Blood is routinely collected in clinic for inhibitor surveillance and these samples were also used to measure factor VIII. A one-stage assay was used for all samples plus a two-stage chromogenic ReFacto standard for patients treated with ReFacto AF (Pfizer).

We assumed that collecting a trough level in clinic would be more achievable and acceptable to patients rather than a full pharmacokinetic study as recommended by UKHCDO [1].

Of the 16 trough samples processed, eight had a trough level $>3 \mathrm{lU} / \mathrm{dL}$. However, two patients in this group had reported breakthrough bleeds and one had recently undergone complex orthopaedic surgery, therefore, only five patients were suitable for dose reduction.

In each of these patients, the factor VIII dose was reduced to the next smallest vial size, if the patient had been using two vials per treatment previously, we attempted to reduce this to a single vial. The doses were reduced by between 4-19 IU/kg in the first instance.

Following dose reduction, patients were encouraged to contact the clinical nurse specialist if they had any questions or concerns. Routine clinic follow-up was arranged for six months; however, some patients were seen by the CNS sooner where requested by the patient. Bleed diaries were reviewed and 48-hour trough samples were repeated.

\section{Results}

No breakthrough bleeds were reported in these five patients. After dose reduction, 48 hour FVIII trough levels ranged from 0.5 to $9 \mathrm{IU} / \mathrm{dL}$, using one-stage assays (Table 1 ). The two-stage chromogenic ReFacto AF standard was $<1 / \mathrm{U} / \mathrm{dL}$.

We observed that of the five patients, two had previously had an inhibitor and have multiple joint arthropathies. These two patients continued to require higher doses of 
factor VIII (19 and $29 \mathrm{IU} / \mathrm{kg}$ ) to maintain a trough of $1-3 \mathrm{IU} / \mathrm{dL}$.

Those who were not eligible for dose reduction were generally the younger patients in the cohort. It is possible that these patients have more active lifestyles, which may influence the consumption of FVIII.

\section{Discussion}

Obtaining an accurate 48-hour trough sample proved to be a challenge for a number of reasons. Patients' lifestyles, work and family commitments often meant that their treatment could not be administered 48 hours prior to sampling. We tried to accommodate this by accepting a sample as a trough if taken 44-52 hours post-infusion. However, it was still not always possible to collect an accurate sample either because the patient had forgotten to adjust his treatment regime and had infused factor within the previous 48 hours, or had not treated for more then 52 hours.

Not all patients on prophylaxis maintain accurate bleed diaries or treatment logs. We have found that for many, the use of the electronic system Haemtrack, has improved data collection. We continue to remind patients to update their records and to inform them of why the information is needed. Some haemophilia centres require treatment records to be submitted monthly, and will withhold home delivery for patients that are not compliant. It would be useful for haemophilia centres to reach a consensus and adopt a common approach as to when it is appropriate to withhold home delivery. At St Georges Healthcare NHS Trust, home delivery is only suspended if a patient has failed to attend two consecutive clinic appointments.

As health care professionals, we accept that our patients with severe haemophilia are experts in their condition. It is important that we work in partnership with patients to identify a treatment regimen that is not only clinically effective and economically viable but also acceptable to the patient. In most cases, factor concentrates are delivered directly to the patient, so clinicians have no direct control over the dose infused at home or the frequency of infusion. We can use home delivery records and home treatment logs to initiate discussion with patients but ultimately prescribed regimens are only recommendations and are difficult to monitor. For this reason it is essential that there is an open and honest dialogue between patients and the haemophilia centre.

Within our patient population, four different factor VIII concentrates are used. Of the five patients in whom dose reduction was achieved, two used the same concentrate and achieved the lowest dosage. It would be interesting to explore whether the concentrate used influences the dose required although this may be influenced by lifestyle factors, as well as age, arthropathy and previous inhibitor history, making direct comparisons difficult.

Nevertheless, we would also like to explore further the use of the chromogenic ReFacto AF standard and onestage assays for patients treated with ReFacto AF. This is the most widely used factor VIII in the UK, and the manufacturer recommends that a ReFacto AF standard chromogenic assay should be used to monitor these patients. At St Georges Healthcare NHS Trust we have been using both assays and comparing results. Discrepant results have been found between the two assays, with the ReFacto AF standard generally giving a trough level of $\mathrm{FVIII<1} \mathrm{IU/dL} \mathrm{[5].} \mathrm{The} \mathrm{one-stage} \mathrm{assay} \mathrm{generally} \mathrm{gives} \mathrm{a}$ higher level and we found in one patient, that we were able to reduce prophylaxis dose (from 37 to $191 \mathrm{U} / \mathrm{kg}$ ) while being guided by the one-stage assay. This fell from 7.4 $\mathrm{IU} / \mathrm{dL}$ to $0.5 \mathrm{IU} / \mathrm{dL}$. The ReFacto AF assay was $<1 \mathrm{IU} / \mathrm{dL}$, however, the patient did not experience any breakthrough bleeding episodes. He is also the youngest patient in this group with the most active lifestyle.

\section{Conclusion}

Within this study, the role of the clinical nurse specialist was vital. The patient generally only has contact with their consultant in clinic every six months. In between these visits, the clinical nurse specialist is able to maintain a dialogue with patients, providing advice, support and reassurance. We found some patients were very anxious about changes to their regimen, being fearful of what they perceived as cheaper, sub-standard regimens. The CNS was able to review trough levels and infusion records in between clinic visits if necessary.

Over 12 months, we estimate that at least $£ 200,000$ was saved in the cost of factor VIII concentrates used by these five patients. Two went on to have further dose reductions as their trough levels remained $>3 \mathrm{lU} / \mathrm{dL}$.

As factor concentrates represent the most expensive

\section{TABLE 1: Study results}

\begin{tabular}{|c|c|c|c|c|c|}
\hline & Patient 1 & Patient 2 & Patient 3 & Patient 4 & Patient 5 \\
\hline Age (years) & 44 & 40 & 48 & 58 & 77 \\
\hline Trough (IU/dL) & 0.5 & 1.5 & 3.4 & 6.1 & 9.0 \\
\hline Breakthrough bleeds & None & None & None & None & None \\
\hline Dose (IU/kg) & 19 & 6 & 19 & 12 & 29 \\
\hline Concentrate & ReFacto Al & Helixate NexGen & Fandhi & Helixate NexGen & Fandhi \\
\hline Remarks & One-stage & & Previous ir & & Previous inhibitor \\
\hline
\end{tabular}


component of haemophilia care within our Trust, implementing personalised prophylaxis regimes could significantly reduce the cost of the service without compromising care. Although this is a very small study our findings could be significant in the context of the economic pressures currently being exerted on NHS expenditure.

\section{Acknowledgements}

The author has no interests that might be perceived as posing a conflict or bias.

\section{References}

1 Richards M, Williams M, Chalmers E et al. A United Kingdom Haemophilia Centre Doctors' Organization guideline approved by the British Committee for Standards in Haematology: quideline on the use of prophylactic factor VIII concentrate in children and adults with severe haemophilia A. Br J Haematology 2010: 149: 498-507.

2 Morfini M. Pharmacokinetics of factor VIII and factor IX. Haemophilia 2003; 9 (Suppl 1): $94-9$

3 Bjorkman S. Prophylactic dosing of factor VIII and factor IX from a clinical pharmacokinetic perspective. Haemophilia 2003; 9 (Suppl. 1): 101-8

4 Collins PW. Personalized prophylaxis. Haemophilia 2012; 18 (suppl. 4): 131-5.

5 Anwar M, Uprichard J, Austin SK. Discrepant one-stage clotting versus chromogenic FVIII assays in haemophilia patients on ReFacto AF. Oral presentation at British Society for Haematology, Annual Scientific Meeting, 2013

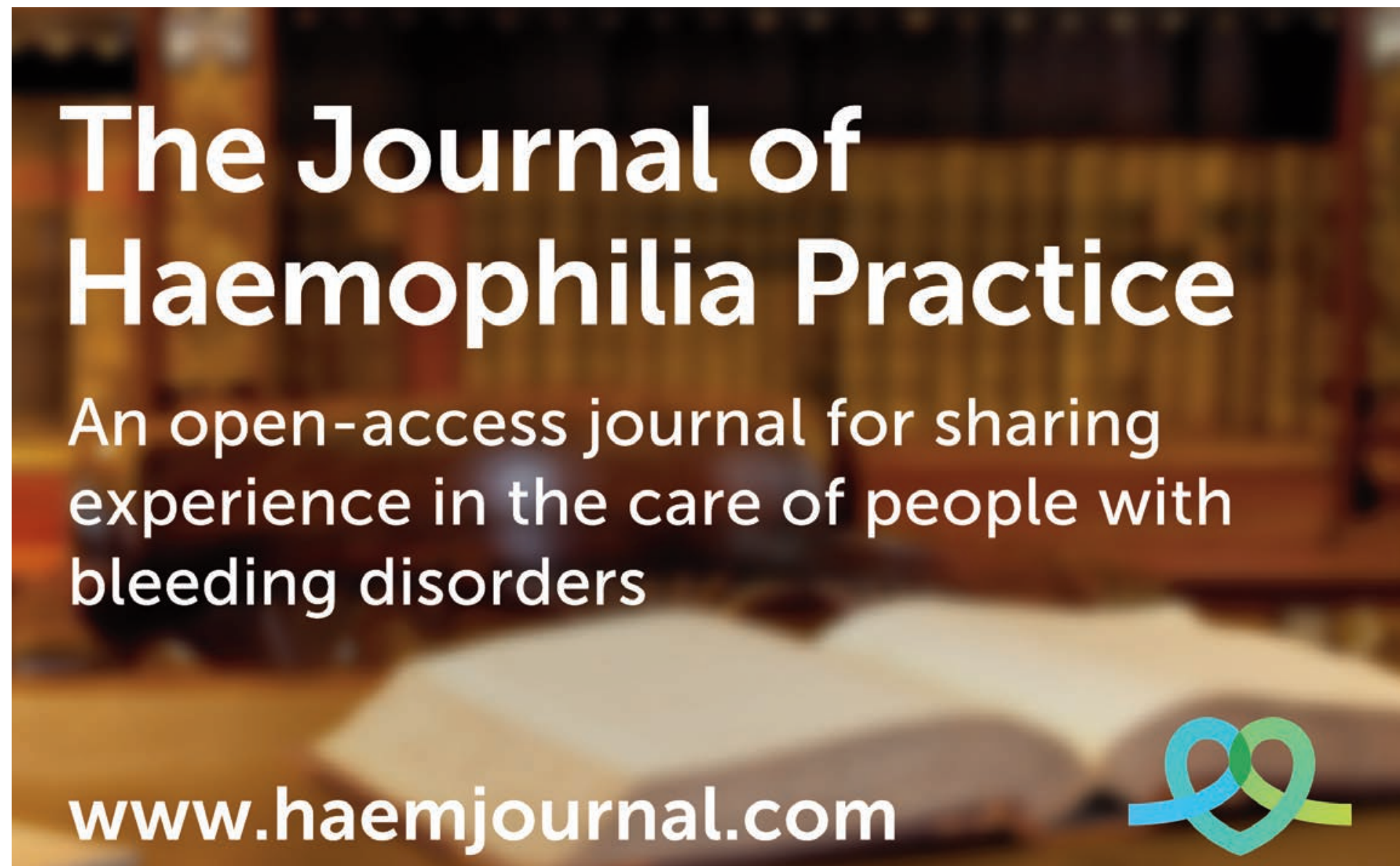

